\title{
THE HOMEOMORPHIC TRANSFORMATION OF $c$-SETS INTO $d$-SETS ${ }^{1}$
}

\section{WILLIAM J. GORMAN III}

Unless otherwise specified, the sets with which we shall deal will be subsets of the open interval $I=(0,1)$. The term $c$-set will be used to describe a set each of whose points is a bilateral condensation point of the set. A $d$-set will be a set that has metric density 1 at each of its points. (Clearly, every $d$-set is a $c$-set, but not conversely.)

In this terminology, a necessary and sufficient condition for a function $f$ to belong to the first Baire class and possess the Darboux (intermediate value) property is that for every real number $a$, the sets

$$
E_{a}=\{x: f(x)<a\} \text { and } E^{a}=\{x: f(x)>a\}
$$

be $F_{\sigma}$ sets [4, Theorem 1$]$. The approximately continuous functions can be characterized in a very similar fashion, namely, by replacing " $c$-sets" by " $d$-sets." (This follows from the definition and the fact that such functions belong to the first Baire class [1, pp. 165 and 181].)

In the light of these two characterizations, the fact that any Baire 1 Darboux function can be transformed by some appropriate homeomorphic change of variable into an approximately continuous function [3] leads to the question: can any $F_{\sigma} c$-set be transformed by some homeomorphism of $I$ on to itself into an $F_{\sigma} d$-set?

The purpose of this paper is to prove that the answer to this question is affirmative and, in so doing, to elucidate something of the structure of $F_{\sigma} c$-sets.

The first lemma is the basis of the structure study.

Lemma 1. For any first category $F_{\sigma} c$-set $C$ having 0 and 1 as limit points, there is a countable collection of sets $Q_{1}, Q_{2}, \cdots$ having the following properties:

(1) $\cup_{i=1}^{\infty} Q_{i}-\{0,1\}=C$;

(2) $Q_{1} \cup \ldots \cup Q_{i}$ is a nowhere-dense perfect set for every positive integer $i$;

(3) $Q_{1}$ contains 0 and 1 ;

(4) for each positive integer $i>1, Q_{i}$ meets the closure of every interval

Received by the editors August 2, 1965.

1 This work constitutes a part of the author's doctoral dissertation. The research was sponsored by the National Science Foundation through Grant GP-3515. The author gratefully acknowledges the guidance of Professor Casper Goffman. 
of $I-\left(Q_{1} \cup \ldots \cup Q_{i-1}\right)$ in a nowhere-dense perfect set that includes both the endpoints of the interval; and

(5) for each positive integer $i>1, Q_{1} \cup \ldots \cup Q_{\imath-1}$ and $Q_{i}$ have no other common points than these endpoints.

Proof. For any set $S$, let (in this proof only) $[S]$ denote the set of condensation points of $S$.

That $C$ is the union of a countable number of nowhere-dense closed sets $F_{1}, F_{2}, \ldots$ is already known.

Although 0 cannot be in $F_{1}$, the fact that $C$ is a $c$-set permits the construction of a sequence $\left\{S_{i}\right\}$ of disjoint closed intervals converging toward 0, each $S_{i+1}$ being located entirely to the left of $S_{i}$, and such that for each $i$ there is an $n(i)$ for which $\left[S_{i} \cap F_{n(i)}\right]$ is uncountable. A similar construction using $T_{i}$ and $m(i)$ as the analogs of $S_{i}$ and $n(i)$ is made for the point 1 . Then, the set

$$
Q_{1}=\left[F_{1}\right] \cup \bigcup_{i=1}^{\infty}\left[S_{i} \cap F_{n(i)}\right] \cup \bigcup_{i=1}^{\infty}\left[T_{i} \cap F_{m(i)}\right] \cup\{0,1\}
$$

is nowhere-dense and perfect. We denote the (open) components of its complement by $I_{1 j}=\left(a_{1 j}, b_{1 j}\right), j=1,2, \cdots$.

Using $F_{2} \cap \bar{I}_{1 j}, S_{1 j k}, n(1 j k), T_{1 i k}$, and $m(1 j k)$ as the analogs of $F_{1}$, $S_{i}, n(i), T_{i}$, and $m(i)$, we perform an identical process on each $\bar{I}_{1 j}$. (If $a_{1 j} \in\left[F_{2} \cap \bar{I}_{1 j}\right]$, the intervals $S_{1 j k}$ are defined to be empty; and similarly if $b_{1 j} \in\left[F_{2} \cap \bar{I}_{1 j}\right]$, the intervals $T_{1 j k}$ are defined to be empty.) Then, we define the set

$$
\begin{aligned}
Q_{2}=\bigcup_{j=1}^{\infty}\left(\left(\left[F_{2}\right] \cap \bar{I}_{1 j}\right) \cup \bigcup_{k=1}^{\infty}\left[S_{1 j k} \cap F_{n(1 j k)}\right]\right. & \cup\left\{a_{1 j}\right\} \\
& \left.\cup \bigcup_{k=1}^{\infty}\left[T_{1 j k} \cap F_{m(1 j k)}\right] \cup\left\{b_{1 j}\right\}\right) ;
\end{aligned}
$$

and we denote the (open) components of $I-\left(Q_{1} \cup Q_{2}\right)$ by $I_{2 j}=\left(a_{2 j}, b_{2 j}\right)$, $j=1,2, \cdots$.

In this manner, we define for each $i>1$ the set

$$
\begin{aligned}
Q_{i}=\bigcup_{j=1}^{\infty}\left(\left(\left[F_{i}\right] \cap \bar{I}_{1 j k}\right) \cup \bigcup_{k=1}^{\infty}\left[S_{i j k} \cap F_{n(i j k)}\right]\right. & \cup\left\{a_{i j}\right\} \\
& \left.\cup \bigcup_{k=1}^{\infty}\left[T_{i j k} \cap F_{m(i j k)}\right] \cup\left\{b_{i j}\right\}\right) .
\end{aligned}
$$

It is easily seen that $Q_{i}$ satisfies (2), (4), and (5). The fact that $C$ is a $c$-set necessitates that each of the points in each of the countable sets 
$\left(F_{1} \cup \ldots \cup F_{i}\right)-\left(Q_{1} \cup \ldots \cup Q_{i}\right)$ be a condensation point of some $F_{k}, k>i$. Hence, (1) is true.

The next lemma shows that there is essentially no topological difference among nowhere-dense $F_{\sigma} c$-sets.

Lemma 2. If $S$ and $T$ are two nowhere-dense $F_{\sigma} c$-sets having 0 and 1 as limit points, there is a homeomorphism $\phi$ of I onto itself such that $T=\phi(S)$.

Proof. Let $P_{1}, P_{2}, \cdots$ and $Q_{1}, Q_{2}, \cdots$ be the collections associated by Lemma 1 with $S$ and $T$, respectively. $I_{i 1}, I_{i 2}, \cdots$ will denote the (open) components of the complement of $P_{1} \cup \ldots \cup P_{i}$.

Every (closed) interval component of $I-S$ is contained in some $I_{1 j}$. Conversely, every $I_{1 j}$ must contain an interval component of $I-S$. (In fact, since $S$ is a $c$-set, each $I_{1 j}$ must contain infinitely many interval components of $I-S$.) For each $j$, let $K_{1 j}$ be such a component of greatest length contained in the (open) interval $I_{1 j}$. Similarly, for each $j$, let $K_{2 j}$ be an interval component of $I-S$ of greatest length contained in $I_{2 j}$. In particular, if $I_{2 j}$ contains some $K_{1 t}$, then $K_{2 j}$ should be chosen to be $K_{1 t}$. In this manner, an interval component $K_{i j}$ of $I-S$ is associated with each $I_{i j}$ in such fashion that:

(1) $K_{i j} \subset I_{i j}$;

(2) there is in $I_{i j}$ no component of $I-S$ of length exceeding that of $K_{i j}$; and

(3) if $I_{\imath j}$ contains some $K_{s t}$, where $s<i$, then $K_{i j}$ is the same as $K_{s t}$.

For any given interval component $K$ of $I-S$, there are only a finite number of components of $I-S$ having length greater than or equal to that of $K$. The nature of the sets $P_{i}$ and the fact that the in terval components of $I-S$ do not abut require that for $i$ sufficiently large $K$ be in a different component of the set $I-\left(P_{1} \cup \ldots \cup P_{i}\right)$ than each of the components of $I-S$ having length greater than or equal to that of $K$. Therefore, the collection of $K_{i j}$ includes all the interval components of $I-S$.

There is a homeomorphism $\phi_{1}$ of $I$ onto itself such that

(1) $\phi_{1}\left(P_{1}\right)=Q_{1}$; and

(2) $\phi_{1}\left(K_{1 j}\right)=L_{1 j}$, a (closed) in terval component of $I-T$ of greatest length contained in $\phi_{1}\left(I_{1 j}\right)$, for all $j$.

Similarly, by working on the closure of each in terval of $I-\left(P_{1} \cup P_{2}\right)$, we can produce a homeomorphism $\phi_{2}$ of $I$ on to itself such that

(1) $\phi_{2}(x)=\phi_{1}(x)$ if $x$ is in $P_{1}$ or in any $K_{1 j}$;

(2) $\phi_{2}\left(P_{2}\right)=Q_{2}$; and 
(3) $\phi_{2}\left(K_{2 j}\right)=L_{2 j}$, an interval component of $I-T$ of greatest length contained in $\phi_{2}\left(I_{2 j}\right)$, for all those $j$ for which $K_{2 j}$ is not identical with some $K_{1 t}$.

In this manner, a sequence $\phi_{i}$ of homeomorphisms is constructed so that for each integer $i>1$,

(1) $\phi(x)=\phi_{i-1}(x)$ if $x$ is in any $P_{k}, k \leqq i-1$, or in any $K_{k j}, k \leqq i-1$;

(2) $\phi_{i}\left(P_{i}\right)=Q_{i}$;

(3) $\phi_{i}\left(K_{i j}\right)=L_{i j}$, an interval component of $I-T$ of greatest length contained in $\phi_{i}\left(I_{i j}\right)$, for all those $j$ for which $K_{i j}$ is not identical with some $K_{s t}, s<i$.

By an argument similar to that given for the collection of $K_{i j}$, the collection of $L_{i j}$ can be shown to contain all the interval components of $I-T$. Thus, for every $i,\left|\phi_{m}(x)-\phi_{n}(x)\right|$ is no greater than the length of the longest component of the complement of

$$
\bigcup_{k=1}^{i-1}\left(Q_{k} \cup \bigcup_{j} L_{k j}\right)
$$

$\left(U_{j}\right.$ indicating union over all $j$ for which $L_{k j}$ has been defined), for all $m, n \geqq i$ and for all $x$. Since the length of the longest of these components approaches 0 as $i$ becomes arbitrarily large, $\phi_{i}$ converges uniformly to a nondecreasing continuous function $\phi$. But, $\phi$ preserves the linear order existing among all the points in all the sets $K_{i j}$, and these points form a dense set. Hence, $\phi$ is strictly increasing and, therefore, a homeomorphism.

Clearly, $T=\phi(S)$.

In the light of this lemma, the proof that any nowhere-dense $F_{\sigma}$ $c$-set can be transformed into a $d$-set is little more than exhibiting a nowhere-dense $F_{\sigma} d$-set-an easy matter. Let $P$ be a nowhere-dense perfect set of measure $1 / 2$. The set of points of $P$ at which $P$ has metric density 1 is also of measure $1 / 2$ and contains an $F_{\sigma}$ set of the same measure. Consequently, this $F_{\sigma}$ set is a $d$-set. Hence, we can state the following lemma.

Lemma 3. If $C$ is any nowhere-dense $F_{\sigma} c$-set, there is a homeomorphism of $I$ onto itself that transforms $C$ into a (nowhere-dense $F_{\sigma}$ ) $d$-set.

We shall now show that there is no topological difference among the first category $F_{\sigma} c$-sets that are dense in $I$.

LemMa 4. If $S$ and $T$ are any two first-category $F_{\sigma} c$-sets that are dense in $I$, there is a homeomorphism $\phi$ of $I$ onto itself such that $T=\phi(S)$.

Proof. The proof is the same as that of Lemma 2 with the following alterations. 
(1) Everything concerned with the $K_{i j}$ and the $L_{i j}$ is deleted.

(2) In proving that $\phi$ is increasing, the dense set of endpoints of the $I_{i j}$ assumes the role of the dense set formed from the $K_{i j}$ in Lemma 2.

Since any $F_{\sigma}$ set of measure 1 contained in the set of irrational points is a dense first-category $d$-set, the following lemma is a simple corollary of Lemma 4.

Lemma 5. If $C$ is any first-category $F_{\sigma} c$-set dense in $I$, there is a homeomorphism of $I$ onto itself that transforms $C$ into a (first-category $\left.F_{\sigma}\right) d$-set of measure 1 .

The main result will be based upon Lemmas 3 and 5. The following result will also be used directly, but in a very minor role.

Lemma 6. If $C$ is an $F_{\sigma} c$-set dense in $I$, then there is in $C a$ nowheredense $F_{\sigma}$ set having 0 and 1 as limit points.

Proof. Since $C$ is an uncountable Borel set, it must contain a (nowhere-dense) perfect set [2]. Hence, there is a homeomorphism $\phi$ of $I$ on to itself such that $\phi(C)$ has positive measure. Let $P$ be any nowhere-dense set having measure greater than that of $I-\phi(C)$. Then, there is an $F_{\sigma} d$-set $F$ contained in $\phi(C) \cap P$. Hence, $\phi^{-1}(F)$ is a nowhere-dense $F_{\sigma}$ set contained in $C$.

Let $a$ and $b$ be, respectively, the left and right extreme limit points of $\phi^{-1}(F)$. If $a>0$, the above type construction is made on $(0, a / 2)$. The process is continued (through possibly denumerably many steps) until 0 is the leftmost limit point of the union of all the $F_{\sigma}$ sets constructed. The procedure if $b<1$ is completely analogous. The union of all the $F_{\sigma}$ sets constructed is the desired subset of $C$.

We are now prepared to prove the main result.

THEOREM. If $C$ is any $F_{\sigma} c$-set in $I$, there is a homeomorphism $\phi$ of $I$ onto itself that transforms $C$ into an $F_{\sigma} d$-set.

Proof. If $C$ is either (1) nowhere-dense or (2) of the first category and dense in the interval defined by the extreme limit points of $C$, then $\phi$ can be obtained easily from Lemma 3 or Lemma 5, respectively.

In any other case, let $A_{1}, A_{2}, \cdots$ be the (closed) in tervals of maximum length in which $C$ is dense; and for each positive integer $i$, let $S_{i}$ be a nowhere-dense $F_{\sigma} c$-set contained in $A_{i}$ and having both the endpoints of $A_{i}$ as limit points (see Lemma 6 ). (Let the superscript ${ }^{\circ}$ denote the interior of a set.) Then the set 


$$
C^{\prime}=\left(C-\bigcup_{i=1}^{\infty} A_{i}^{\circ}\right) \cup \bigcup_{i=1}^{\infty} S_{i}
$$

is easily seen to be a nowhere-dense $F_{\sigma}$ set contained in $C$. By Lemma 2 , there is a homeomorphism $\phi_{1}$ such that $\phi_{1}\left(C^{\prime}\right)$ is a (nowhere-dense) $F_{\sigma} d$-set.

Let $T_{i}$ denote $\phi_{1}\left(C \cap A_{i}^{\circ}\right)$ if this set is of the first category. If this set is of the second category, let $T_{i}$ be the set formed from it by replacing the interior of each interval component by any first category $F_{\sigma} c$-set dense in that component. Thus, $T_{i}$ is, in every case, a first category $F_{\sigma} c$-set dense in $\phi_{1}\left(A_{i}\right)$. On each of the intervals $\phi_{1}\left(A_{i}\right)$, define the function $\phi_{2}$ to be the analog of the homeomorphism of Lemma 5; and let $\phi_{2}(x)=x$ elsewhere. Finally, let $\phi$ be the homeomorphism $\phi_{2} \circ \phi_{1}$. Since the measure of $\phi\left(T_{i}\right)$ is equal to the measure of $\phi\left(A_{i}\right)$ for every $i, \phi(C)$ is an $F_{\sigma} d$-set.

The extension of this theorem to the entire real line should be rather clear. However, the extension to any other Euclidean space, even to the plane, is a very much open problem; and the first step toward its solution must be the formulation of a suitable definition of $c$-set in the space.

\section{REFERENCES}

1. A. Denjoy, Sur les fonctions derivée sommables, Bull. Soc. Math. France 43 (1915), 161-248.

2. H. Hahn, Reele Funktionen, Chelsea, New York, 1948; p. 356.

3. Isiah Maximoff, Sur la transformation continue de quelque fonctions en derivées exactes, Bull. Phys.-Math. Kazan (3) 12 (1940), 57-81. (Russian. French summary)

4. C. J. Neugebauer, Darboux functions of Baire class one and derivatives, Proc. Amer. Math. Soc. 13 (1962), 838-843.

Purdue University 